\title{
AN IMPLICIT, NUMERICAL METHOD FOR SOLVING THE TWO-DIMENSIONAL HEAT EQUATION*
}

\author{
BY \\ GEORGE A. BAKER, Jr. \\ AND \\ THOMAS A. OLIPHANT \\ Los Alamos Scientific Laboratory, University of California, Los Alamos, New Mexico
}

1. Introduction. We develop an implicit scheme for the numerical solution of the two-dimensional heat-flow problem. In the linear case we are able to solve exactly the full two-dimensional set of implicit equations. This solution is possible because we choose a difference scheme for which the equations are factorable into two one-dimensional sets. This factorability is basic to the method.

We extend this method to non-linear equations and non-rectangular regions by the use of an iterative scheme to solve the implicit equations obtained. This scheme provides second-order convergence, and in the cases we have tested only a very few iterations per time step were required.

The method is proved to be unconditionally stable both in the linear and non-linear cases. (We consider only a special set of non-linear problems in the stability analysis.) We prove stability in the linear case by the usual type of Fourier analysis and superposition of solutions. In the non-linear case we show that the norms of the solutions of the difference equation with homogeneous boundary conditions tend to zero as time tends to infinity. This method is limited in mesh size and time-step length only by the requirements of accuracy and not stability.

By way of illustration we include a discussion of two numerical examples.

2. The linear case. The basic partial differential equation which we wish to consider is

$$
\frac{\partial^{2} \theta}{\partial x^{2}}+\frac{\partial^{2} \theta}{\partial y^{2}}=\alpha^{-2} \frac{\partial \theta}{\partial t}
$$

where $x$ and $y$ are space coordinates, $t$ is time, and $\alpha$ is a constant. We wish to approximate this differential equation by a finite difference scheme to allow the approximate numerical calculation of the function $\theta$. If we denote spatial points by either the pair of indices $(i j)$ or the pair $(k l)$, and time by $n$, then we may write, using the Einstein summation convention, a general, linear difference scheme as

$$
B_{k l}^{i j}{ }^{n} \theta_{i i}={ }^{n} a_{k l},
$$

where the ${ }^{n} a_{k l}$ depend on quantities with time earlier than $n$. We wish to choose a particular $B_{k l}^{i j}$ which will both represent (2.1) and allow for easy calculation of ${ }^{n} \theta_{k l}$ for successive values $n$. Clearly, an explicit differencing scheme $\left(B_{i j}^{k l} \alpha \delta_{i}^{k} \delta_{i}^{l}\right.$, where $\delta_{i}^{k}$ is the Kronecker delta) has both these properties, however, such a scheme is well known to have the disadvantage of being only conditionally stable. Implicit schemes are usually

*Received August 25, 1958. Work performed under the auspices of the U. S. Atomic Energy Commission. 
unconditionally stable. One such implicit scheme is the Douglas-Peaceman alternatingdirection method [1]. It represents the application of the one-dimensional Bruce, Peaceman, Rachford, and Rice [2] method to two dimensions. It has the disadvantage that only one direction is treated exactly at each advance in time. We feel it desirable to choose a differencing scheme which will permit an exact treatment over the entire two dimensional mesh. With this view in mind we note that if we may factor $B_{k l}^{i i}$ as

$$
B_{k l}^{i j}=A_{k}^{i} B_{l}^{i}
$$

then we may triangularly resolve $A_{k}^{i}$ and $B_{l}^{i}$ separately as was done in one dimension by Bruce, Peaceman, Rachford, and Rice and obtain a method for the direct calculation of the ${ }^{n} \theta_{k l}$ from ${ }^{m} \theta_{k l}$ with $m<n$. We shall now restrict ourselves to 9 -point difference schemes, i.e.,

$$
B_{k l}^{i j}=0 \text { if }|i-k|>1 \text {, or }|j-l|>1 .
$$

It will be shown later that 5 -point difference schemes are unfactorable. Thus let $A_{k}^{i}$ and $B_{l}^{i}$ be triple-diagonal matrices,

$$
\begin{aligned}
& A_{k}^{i}=\left(x_{1} \delta_{k}^{i+1}+x_{2} \delta_{k}^{i}+x_{3} \delta_{k}^{i-1}\right), \\
& B_{l}^{i}=\left(y_{1} \delta_{l}^{i+1}+y_{2} \delta_{l}^{i}+y_{3} \delta_{l}^{i-1}\right) .
\end{aligned}
$$

In order to represent Eq. (2.1), $B_{k l}^{i i}$ must be a numerical representation of the Laplacian plus whatever part of the representation of the time derivative involves $\theta$ at the advanced time. Thus (suppressing the superscript $n$ ),

$$
B_{k l}^{i j} \theta_{i j} \text { represents } \beta \theta_{k l}+\nabla^{2} \theta_{k l} \text {. }
$$

From the symmetry properties of the Laplacian, we may restrict the values of $B_{k l}^{i i}$ (allowing a different mesh-spacing in the $x$ and $y$ directions) to

$$
\begin{aligned}
& B_{k l}^{k+1, l}=B_{k l}^{k-1, l}=\eta, \\
& B_{k l}^{k, l+1}=B_{k l}^{k, l-1}=\lambda, \\
& B_{k l}^{k+1, l+1}=B_{k l}^{k+1, l-1}=B_{k l}^{k-1, l+1}=B_{k l}^{k-1, l-1}=\mu, \\
& B_{k l}^{k l}=\xi .
\end{aligned}
$$

Expanding (2.3) by use of (2.5) we get from (2.7)

$$
\begin{aligned}
\eta & =x_{3} y_{2}=x_{1} y_{2} \\
\lambda & =y_{1} x_{2}=y_{3} x_{2}, \\
\mu & =x_{1} y_{1}=x_{3} y_{1}=x_{1} y_{3}=x_{3} y_{3}, \\
\xi & =x_{2} y_{2} .
\end{aligned}
$$

These nine equations in six unknowns imply the restrictions

$$
\begin{gathered}
\eta \lambda=\mu \xi, \\
x_{1}=x_{3}, \quad y_{1}=y_{3} .
\end{gathered}
$$

It should be noted that if $\mu$ is zero, as for a five-point scheme, then by (2.9) either $\lambda$ or $\eta$ must also be zero so that no factorable five-point scheme is possible. 
More generally it can be shown that if we let

$$
B_{k l}^{i i}=w_{k l}^{m n} m_{m n}^{i i}
$$

with $w_{k l}^{m n}=0$ when $k-m>1, k-m<0, l-n>1$, or $l-n<0$ and $b_{m n}^{i j}=0$ when $i-m>1, i-m<0, j-n>1$, or $j-n<0$, then either (2.9) or $\xi=4 \mu$ must hold.

To obtain a proper representation of the Laplacian, we consider the Taylor series expansion of $\theta$,

$$
\begin{aligned}
\theta(x+\Delta x, y+\Delta y)=\theta(x, y) & +b \Delta x+c \Delta y+d(\Delta x)^{2}+e(\Delta x)(\Delta y)+f(\Delta y)^{2} \\
& +g(\Delta x)^{3}+h(\Delta x)^{2} \Delta y+i(\Delta x)(\Delta y)^{2}+j(\Delta y)^{3} .
\end{aligned}
$$

Applying the difference scheme (2.7) to (2.11) we obtain

$$
B_{k l}^{i j} \theta_{i i}=(4 \mu+2 \eta+2 \lambda+\xi) \theta_{k l}+(4 \mu+2 \eta) d(\Delta x)^{2}+(4 \mu+2 \lambda) f(\Delta y)^{2} .
$$
As

$$
\frac{\partial^{2} \theta}{\partial x^{2}}+\frac{\partial^{2} \theta}{\partial y^{2}}=2(d+f)
$$

we must have, by (2.6),

$$
\begin{gathered}
(2 \mu+\eta)(\Delta x)^{2}=1, \\
(2 \mu+\lambda)(\Delta y)^{2}=1, \\
4 \mu+2 \eta+2 \lambda+\xi=\beta .
\end{gathered}
$$

Solving (2.9) and (2.14) we obtain

$$
\begin{gathered}
\mu=1 /\left[\beta(\Delta x)^{2}(\Delta y)^{2}\right], \\
\eta=(\Delta x)^{-2}\left\{1-2 /\left[\beta(\Delta y)^{2}\right]\right\}, \\
\lambda=(\Delta y)^{-2}\left\{1-2 /\left[\beta(\Delta x)^{2}\right]\right\}, \\
\xi=\beta\left\{1-2 /\left[\beta(\Delta y)^{2}\right]\right\}\left\{1-2 /\left[\beta(\Delta x)^{2}\right]\right\} .
\end{gathered}
$$

We may now solve (2.8) and (2.15) for $x_{i}$ and $y_{i}$. Thus,

$$
\begin{aligned}
B_{k l}^{i j}=\left[\beta(\Delta x)^{2}(\Delta y)^{2}\right]^{-1}\left\{\delta_{k}^{i+1}+\left[\beta(\Delta x)^{2}-2\right]\right. & \left.\delta_{k}^{i}+\delta_{k}^{i-1}\right\} \\
& \times\left\{\delta_{l}^{i+1}+\left[\beta(\Delta y)^{2}-2\right] \delta_{l}^{i}+\delta_{l}^{i-1}\right\} .
\end{aligned}
$$

Or,

$$
B_{k l}^{i j}=\left[\beta(\Delta x)^{2}(\Delta y)^{2}\right]^{-1} A_{k}^{i} B_{l}^{i},
$$

where, letting

$$
\begin{aligned}
& u=\beta(\Delta x)^{2}-2, \\
& v=\beta(\Delta y)^{2}-2,
\end{aligned}
$$


we define

and

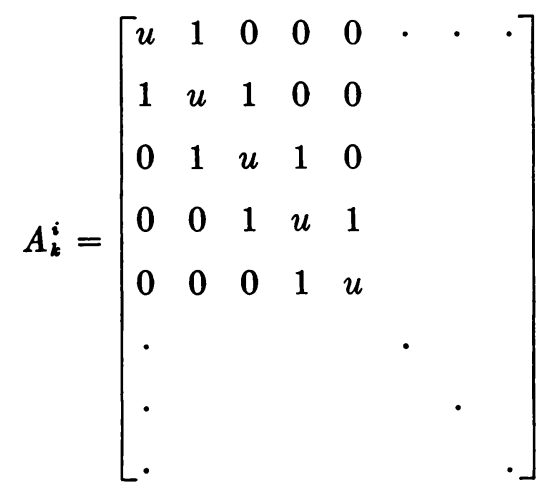

$$
B_{l}^{i}=\left[\begin{array}{llllllll}
v & 1 & 0 & 0 & 0 & \cdot & \cdot & . \\
1 & v & 1 & 0 & 0 & & & \\
0 & 1 & v & 1 & 0 & & \\
0 & 0 & 1 & v & 1 & & \\
0 & 0 & 0 & 1 & v & & & \\
\cdot & & & & & . & & \\
\cdot & & & & & & . & \\
. & & & & & & &
\end{array}\right] .
$$

We may factor $A_{k}^{i}$ and $B_{l}^{i}$ as follows:

$$
\begin{aligned}
& A_{k}^{i}={ }_{x} w_{k}^{m}{ }_{x} b_{m}^{i}=\left[\begin{array}{ccccccc}
u-s_{0} & 0 & 0 & 0 & 0 & \cdot & \cdot \\
1 & u-s_{1} & 0 & 0 & 0 & \\
0 & 1 & u-s_{2} & 0 & 0 & \\
0 & 0 & 1 & u-s_{3} & 0 & \\
0 & 0 & 0 & 1 & u-s_{4} & \\
\cdot & & & & & \cdot & \\
\cdot & & & & & & \\
\cdot & & & & & &
\end{array}\right] \\
& \times\left[\begin{array}{cccccccc}
1 & s_{1} & 0 & 0 & 0 & \cdots & \cdot \\
0 & 1 & s_{2} & 0 & 0 & & \\
0 & 0 & 1 & s_{3} & 0 & & \\
0 & 0 & 0 & 1 & s_{4} & & \\
0 & 0 & 0 & 0 & 1 & & \\
\cdot & & & & & \cdot & \\
\cdot & & & & & & \\
. & & & & & & &
\end{array}\right]
\end{aligned}
$$


where

$$
s_{0}=0 \quad s_{k}=\left(u-s_{k-1}\right)^{-1}
$$

and

$$
\begin{aligned}
& B_{l}^{i}={ }_{\nu} w_{l}^{m}, b_{m}^{j}=\left[\begin{array}{cccccccc}
v-r_{0} & 0 & 0 & 0 & 0 & \cdot & \cdot \\
1 & v-r_{1} & 0 & 0 & 0 & & \\
0 & 1 & v-r_{2} & 0 & 0 & & \\
0 & 0 & 1 & v-r_{3} & 0 & & \\
0 & 0 & 0 & 1 & v-r_{4} & & \\
\cdot & & & & & . & \\
\cdot & & & & & & .
\end{array}\right] \\
& {\left[\begin{array}{cccccccc}
1 & r_{1} & 0 & 0 & 0 & \cdots & \cdot & \\
0 & 1 & r_{2} & 0 & 0 & & \\
0 & 0 & 1 & r_{3} & 0 & & \\
0 & 0 & 0 & 1 & r_{4} & & \\
0 & 0 & 0 & 0 & 1 & & \\
\cdot & & & & & \cdot & \\
\cdot & & & & & & \\
. & & & & & & & \\
.
\end{array}\right]}
\end{aligned}
$$

where

$$
r_{0}=0 \quad r_{l}=\left(v-r_{l-1}\right)^{-1} \text {. }
$$

If we now define

$$
{ }^{n} g_{v \omega}={ }_{x} b_{v}^{i}{ }_{\nu} b_{\omega}^{i n} \theta_{i i}
$$

and

$$
w_{k l}^{v \omega}={ }_{x} w_{k y}^{v} w_{l}^{\omega}
$$

then (2.2) becomes, by (2.17), (2.21), and (2.23),

$$
w_{k l}^{v \omega n} g_{v \omega}=\beta(\Delta x)^{2}(\Delta y)^{2}{ }^{n} a_{k l} \text {. }
$$

Now as $w_{k l}^{v \omega}$ involves only values of $(v \omega)$ such that $v \leq k, \omega \leq l$, we may proceed from low index numbers to high ones and solve (2.27) for the " $g_{v \infty}$ by straightforward elimination. Then as ${ }_{x} b_{v}^{i} b_{\omega}^{i}$ involves only values of $(i j)$ such that $i \geq v, j \geq \omega$, we may proceed from high index numbers to low ones and solve (2.25) for the ${ }^{n} \theta_{i j}$ by straightforward elimination. The formulae involved may be written conveniently as

$$
{ }^{n} g_{i i}=\frac{\beta(\Delta x)^{2}(\Delta y)^{2}{ }^{n} a_{i j}-{ }^{n} g_{i-1, i-1}-\left(v-r_{i-1}\right){ }^{n} g_{i-1, i}-\left(u-s_{i-1}\right){ }^{n} g_{i i-1}}{\left(v-r_{i-1}\right)\left(u-s_{i-1}\right)},
$$




$$
{ }^{n} \theta_{i j}={ }^{n} g_{i j}-s_{i}{ }^{n} \theta_{i+1, i}-r_{j}{ }^{n} \theta_{i, j+1}-s_{i} r_{i}{ }^{n} \theta_{i+1, i+1}
$$

with appropriate modifications at the boundaries.

3. Generalization to the non-linear case. In this section we shall consider generalizing the method given in Sec. 2 to the solution of non-linear partial differential equations of the type

$$
\frac{\partial^{2} \psi}{\partial x^{2}}+\frac{\partial^{2} \psi}{\partial y^{2}}=h(x, y, \psi) \frac{\partial \psi}{\partial t}
$$

In the linear case we modified the equation under consideration by adding $\beta \psi$ to each side. Here we obtain

$$
\beta \psi+\nabla^{2} \psi=\beta \psi+h(x, y, \psi) \frac{\partial \psi}{\partial t}
$$

In the linear case we chose $\beta$ so that the right-hand side was independent of $\theta$ at the advanced time. Here this is not possible. Let us, however, choose

$$
0>\beta \geq-\lambda \quad \underset{i, j}{\operatorname{Minimum}}\left\{h\left(x_{i}, y_{i}, \psi_{i j}\right)+\left.\psi_{i j} \frac{\partial h}{\partial \psi}\right|_{i i}\right\},
$$

where $\lambda$ is the coefficient of $\psi$ at the advanced time in the representation of the time derivative. In the heat flow problem $h$ will be positive and in most cases of interest (see Sec. 6) will vary roughly like $\psi^{-\gamma}$, where $0 \leq \gamma<1$. Let us now guess a value of $\psi$, and substitute it in the right side of the difference equation approximation to (3.2). We may now calculate by the method of Sec. 2 the values of $\psi_{i j}$ which appear on the left side. If our guess was close to the true solution, we may expand everything to first order in the error. Let

$$
\begin{aligned}
& \psi_{\text {guess }}=\psi_{\text {true }}+\epsilon^{*} \\
& \psi_{\text {calculated }}=\psi_{\text {true }}+\epsilon .
\end{aligned}
$$

Then, by (3.2)

$$
\beta \epsilon_{i j}+\nabla^{2} \epsilon_{i j}=\left[\beta+\lambda h(i j)+\left.\lambda \Delta_{i j} \frac{\partial h}{\partial \psi}\right|_{i j}\right] \epsilon_{i j}^{*},
$$

where $\lambda \Delta_{i j}$ is the representation of the time derivative. Let us restrict the possible choice of representations of the time derivative such that (all $\psi \geq 0$ )

$$
\left({ }^{n} \Delta_{i j}\right) /\left({ }^{n} \psi_{i i}\right) \leq 1
$$

This condition will be satisfied if, for instance,

$$
{ }^{n} \Delta_{i j}={ }^{n} \psi_{i j}-{ }^{n-1} \psi_{i j}
$$

and it is satisfied for our choice (4.1) as long as the temperature does not drop by a factor of more than 4 at one time step. We shall neglect $\nabla^{2} \epsilon_{i j}$ as small in (3.5) because we expect our guess to have only small systematic errors, rather than the type of errors which would create large errors in the second derivative. Thus,

$$
\epsilon_{i j} \approx\left\{1+\left[\lambda h(i j)+\left.{ }^{n} \Delta_{i j} \frac{\partial h}{\partial \psi}\right|_{i j}\right] / \beta\right\} \epsilon_{i j}^{*}=-\chi_{i j} \epsilon_{i j}^{*} .
$$


Because of our selection (3.3) of $\beta, \chi$ must be positive or zero. Therefore, if we take a weighted average of $\psi_{\text {guess }}$ and $\psi_{\text {cal oulated }}$ as

$$
\psi^{\prime}=\frac{\psi_{\text {cal culsted }}+\chi \psi_{\text {guess }}}{1+\chi},
$$

then $\psi^{\prime}$ will agree with $\psi_{\text {true }}$ to within second order in $\epsilon^{*}$. If $\psi^{\prime}$ is now used as a new guess, we may continue our iteration procedure and be assured of second-order convergence. We shall see in Sec. 6 that this scheme provides very rapid convergence in a sample case.

4. Stability. Let us first consider the stability of our method as described in Sec. 2 for the case of linear flow. We pick a representation of $\partial \theta / \partial t$ which, to within terms of third order, gives $\partial \theta / \partial t$ evaluated at the advanced time, namely,

$$
\left(1.5 \theta^{n}-2 \theta^{n-1}+0.5 \theta^{n-2}\right) / \Delta t .
$$

From (2.1) and (2.6) we see that $\beta$ of Sec. 2 is

$$
\beta=-3 /\left(2 \alpha^{2} \Delta t\right) .
$$

Let us obtain the exact solutions to our difference equations. We may expand any function on the mesh points in a Fourier series, so it will be sufficient to consider the behavior of

$$
{ }^{n} \theta_{i j}=\theta_{0} \exp \left(i b_{1} x_{i}+i b_{2} y_{i}\right) X(n) .
$$

We shall assume that

$$
X(n)=Z^{t / \Delta t} .
$$

Substituting (4.3) into (2.1) as expressed by (2.16) we obtain, making use of trigonometric half-angle formulae,

$$
\begin{aligned}
\Gamma & \equiv\left[-\beta(\Delta x)^{2}+4 \sin ^{2}\left(\frac{1}{2} b_{1} \Delta x\right)\right]\left[-\beta(\Delta x)^{2}+4 \sin ^{2}\left(\frac{1}{2} b_{2} \Delta y\right)\right](\beta \Delta x \Delta y)^{-2} \\
& =\left(4 Z^{-1}-Z^{-2}\right) / 3 .
\end{aligned}
$$

Thus,

$$
Z_{ \pm}^{-1}=2 \pm(4-3 \Gamma)^{1 / 2} .
$$

By definition, as $b_{1}$ and $b_{2}$ are real, $\Gamma>1$. Thus, for $\Gamma \leq 4 / 3, Z_{\star}$ are both real and

$$
1>Z_{-} \geq \frac{1}{2} \geq Z_{+}>\frac{1}{3} \text {. }
$$

If $\Gamma>4 / 3$, then $Z_{\star}$ are both complex and

$$
\left|Z_{\star}\right|=(3 \Gamma)^{-1 / 2}<\frac{1}{2} .
$$

Therefore as $\left|Z_{*}\right|<1$, the difference scheme is unconditionally stable.

In the limit as $\Delta x, \Delta y$, and $\Delta t$ tend to zero,

$$
\begin{aligned}
& \left(Z_{-}\right)^{t / \Delta t} \rightarrow \exp \left[-\alpha^{2}\left(b_{1}^{2}+b_{2}^{2}\right) t\right], \\
& \left(Z_{+}\right)^{t / \Delta t} \rightarrow 3^{-t / \Delta t} \exp \left[\frac{1}{3} \alpha^{2}\left(b_{1}^{2}+b_{2}^{2}\right) t\right] .
\end{aligned}
$$

The root $Z_{-}$represents the analytic solution of (2.1). The $\operatorname{root} Z_{+}$enables us to represent any computational error involved in advancing from time $n-2$ to $n-1$. We see from (4.7) and (4.8) that this error is damped by at least a factor of 2 at each time step. 
The behavior of the solution of the difference equations in the limit as $t \rightarrow \infty$ with $\Delta t \rightarrow 0$ is also of interest. This behavior may be obtained by setting $Z=1$ in (4.5) and removing the reality requirement on $b_{1}$ and $b_{2}$. Thus, for the steady state

$$
(\Delta x)^{2}\left[4 \sin ^{2}\left(\frac{1}{2} b_{1} \Delta x\right)\right]^{-1}+(\Delta y)^{2}\left[4 \sin ^{2}\left(\frac{1}{2} b_{2} \Delta y\right)\right]^{-1}=-2 \alpha^{2} \Delta t / 3 .
$$

If we have enough mesh points so that $\Delta x b_{1} \ll 1, \Delta y b_{2} \ll 1$, then we may expand the sines to first order and by some manipulation obtain

$$
\left(b_{1}^{2}+b_{2}^{2}\right) / b_{1}^{2} \approx 2 b_{1}^{2} \alpha^{2} \Delta t /\left(3+2 b_{1} \alpha^{2} \Delta t\right) .
$$

In the differential equation, the steady state solution is characterized by

$$
b_{1}^{2}+b_{2}^{2}=0 \text {. }
$$

In order to get a good steady solution we see from (4.11) and (4.12) that $b_{1}^{2} \alpha^{2} \Delta t$ must be small. In order to get a good time-dependent solution we must be near the limit given in (4.9). This requires that $b_{1} \Delta x$ and $b_{z} \Delta y$ be small, and $b_{1}^{2} \alpha^{2} \Delta t$ be small. Hence, we will get a good asympotic solution when the requirements are met for a good time-dependent solution. We see from the above analysis that this method is limited in mesh size and time-step length only by the requirements of accuracy and not by stability.

In the non-linear case, the analysis is more difficult but proceeds in a similar manner. We shall not investigate stability in the general case (3.1) but shall restrict $h$ to be of the form

$$
h(x, y, \psi)=f(x, y) \psi^{\gamma},
$$

where $0 \geq \gamma>-2$. We shall for convenience consider only the case of homogeneous boundary conditions. We can, of course, simulate non-homogeneous boundary conditions by letting $f$ be very large near the boundary and so make a region near the boundary an effective heat reservoir. In this analysis we follow Bellman [3] and extend his analysis to the non-linear case. We remark that it is both necessary and sufficient for stability in the usual sense that

$$
\operatorname{limit}_{n \rightarrow \infty} \sum_{i, j} h\left(i, j,{ }^{n} \psi_{i j}\right)\left({ }^{n} \psi_{i i}\right)^{2}=0
$$

hold for any initial conditions and homogeneous boundary conditions. We shall prove that our differencing scheme is unconditionally stable in the sense that (4.14) holds for any $\left[\Delta t /(\Delta x)^{2}\right]$.

Let us fix our attention on a time, which we shall denote by $n$. Let us attempt to separate variables. From (2.17) and (3.1) we obtain the equation which must be satisfied for some separable component, $v$. It is

$$
\begin{aligned}
\left\{\left[\beta(\Delta x)^{2}(\Delta y)^{2}\right]^{-1} A_{k}^{i} B_{l}^{i}-\beta \delta_{k}^{i} \delta_{l}^{i}\right\}{ }^{n} \psi_{i j}(v)= & h\left(k, l,{ }^{n} \psi_{k l}\right){ }^{n} \psi_{k l}(v) \\
& \times\left[3-4 Z^{-1}(v)+Z^{-2}(v)\right] /(2 \Delta t),
\end{aligned}
$$

where we assume that

$$
{ }^{n} \psi_{i j}(v)=Z(v)^{n-1} \psi_{i j}(v)
$$

and that

$$
{ }^{n} \psi_{i j}=\sum_{v}{ }^{n} \psi_{i j}(v) p(v)
$$


In fixing our attention on a time $n$ we imagine that ${ }^{n} \psi_{i i}$ is somehow known. If we set

$$
\left[3-4 Z^{-1}(v)+Z^{-2}(v)\right] /(2 \Delta t)=\lambda
$$

then it is easy to show that the solution of (4.15) is the same as the solution of the following problem. Let (suppressing the $n$ and $v$ )

$$
\begin{aligned}
F \equiv \sum_{k, l}\left[\left(\frac{\psi_{k+1, l}-\psi_{k l}}{\Delta x}\right)^{2}+\left(\frac{\psi_{k, l+1}-\psi_{k l}}{\Delta y}\right)^{2}\right. & \\
& \left.-\frac{\left(\psi_{k+1, l+1}-\psi_{k+1, l}-\psi_{k, l+1}+\psi_{k l}\right)^{2}}{\beta(\Delta x)^{2}(\Delta y)^{2}}\right]
\end{aligned}
$$

and

$$
G \equiv \sum_{k, l} h\left(k, l, \psi_{k l}\right)\left(\psi_{k l}\right)^{2}=1 .
$$

Find the extrema of $F+\lambda G$ subject to (4.20) and homogeneous boundary conditions. If we have $N \times M$ interior mesh points, then the theory of quadratic forms [4] assures us, as $\beta$ is negative, that there exist $N M$ orthogonal vectors $\left[{ }^{n} \psi_{k l}(v)\right]$ which satisfy (4.19) and (4.20) and therefore (4.15). The orthogonality is in the sense that

$$
\left({ }^{n} \psi_{k l}(v),{ }^{n} \psi_{k l}(\mu)\right) \equiv \sum_{k, l} h\left(k, l,{ }^{n} \psi_{k l}\right){ }^{n} \psi_{k l}(v){ }^{n} \psi_{k l}(\mu)=\delta_{v \mu} .
$$

As $\lambda$ can be shown to be the negative of $F$, we must have

$$
\lambda<0 .
$$

We may now solve (4.18) for the corresponding values of $Z_{\star}(v)$. Equation (4.18) implies that for the $\Gamma$ of $(4.5)$ we get

$$
\Gamma=1-2 \lambda \Delta t / 3>1
$$

by (4.22). Thus $\left|Z_{-}\right|<1$ as in the linear case. Let us now expand ${ }^{n} \psi_{i j}$ in terms of our set of orthogonal vectors $\left[{ }^{n} \psi_{i j}(v)\right]$. In order to reproduce ${ }^{n-1} \psi_{i j}$ and ${ }^{n-2} \psi_{i j}$ it will be necessary to use parts proportional to $Z_{+}$and to $Z_{-}$; however, we have enough freedom to effect this decomposition as ${ }^{n} \psi_{i j}$ is assumed to be calculated by our difference scheme from the values at the two previous times. Let us now compute the time derivative of the Norm of ${ }^{n} \psi_{i i}$

$$
\begin{aligned}
\frac{\partial}{\partial t}\left\{\sum_{k, l} h\left(k, l,{ }^{n} \psi_{i j}\right)\left({ }^{n} \psi_{i i}\right){ }^{2}\right\}=\sum_{k l} f(k, l) \frac{\partial}{\partial t}\left({ }^{n} \psi_{i j}\right)^{2+\gamma} & \\
& =(2+\gamma) \sum_{k, l} h\left(k, l,{ }^{n} \psi_{k l}\right){ }^{n} \psi_{k l} \frac{\partial^{n} \psi_{k l}}{\partial t} .
\end{aligned}
$$

If we now approximate $\partial \psi / \partial t$ by an expression of the form of (4.1) and use our expansion in terms of ${ }^{n} \psi_{i j}(v)$, then, if $p(v)$ denotes the coefficient of ${ }^{n} \psi_{i j}(v)$ in ${ }^{n} \psi_{i i}$, we get

$\frac{\partial}{\partial t} \operatorname{Norm} \quad\left({ }^{n} \psi_{i j}\right)=(2+\gamma) \sum_{v}|p(v)|^{2} \lambda(v)$

$$
\leq \operatorname{Max}_{v}[\lambda(v)](2+\gamma) \text { Norm }\left({ }^{n} \psi_{i j}\right) .
$$

We must now examine the behavior of $\operatorname{Max}_{v}[\lambda(v)]$. First let us ccnsider the maximum attained by $\operatorname{Max}_{v}[(\lambda(v)]$ with respect to all variations of components for a fixed Norm. 
As this represents continuous variation over a closed and bounded set, the maximum of $\operatorname{Max}_{v}[\lambda(v)]$ must be attained at some point of the set by Weierstrass' theorem. However, applying the arguments that lead to (4.22) we see that for fixed Norm,

$$
\lambda(v) \leq \lambda(\text { Norm })<0 .
$$

We may consider a variation in Norm by simply scaling all the $\psi_{k l}$. It easily follows from (4.19) and (4.20) that for any Norm

$$
\lambda(v) \leq \lambda_{0}\left[\operatorname{Norm}\left({ }^{n} \psi_{i j}\right)\right]^{-\gamma} .
$$

Thence, by (4.25) and (4.27) we have

$$
\frac{\partial}{\partial t}\left[\operatorname{Norm}\left({ }^{n} \psi_{i j}\right)\right] \leq \lambda_{0}(2+\gamma)\left[\operatorname{Norm}\left({ }^{n} \psi_{i j}\right)\right]^{1-\gamma} .
$$

Thus as $0 \geq \gamma>-2$, and $\lambda_{0}<0$, we see that the Norm must decrease to zero. In the linear case $(\gamma=0)$ this decrease is exponential. This proof is subject only to the proviso that $\Delta t$ be small enough so that (4.1) will give us an approximation of the time derivative of the Norm that reproduces the sign properly. As the norm tends to zero, we see that our scheme is unconditionally stable, at least for this special class of problems. It should be noted that both the diffusion equation [2] and the radiation transport equation with power-law, temperature-dependent opacity belong to this class.

Analogous arguments for the non-linear case corresponding to the arguments involving (4.10) to (4.12) indicate that the requirements for obtaining good time-dependent and steady-state solutions are about the same as the requirements for obtaining such solutions in the linear case. Therefore, the non-linear case has the same properties so far as stability and accuracy are concerned as the linear case. The only way in which the numerical solution to the non-linear case differs essentially from the linear case in rectangular coordinates is that an iterative procedure must be used on each time-step in the non-linear case.

5. Extension to non-rectangular regions and three-dimensional problems with an axis of symmetry. Consider a simply-connected, two-dimensional region with more than one boundary point. Riemann's theorem [5] implies that we may map it by a one-to-one conformal transformation into a rectangular region. This transformation amounts to a change of independent variables. If

$$
u=u(x, y) \quad v=v(x, y)
$$

is such a conformal mapping, then (3.1) is transformed [6] into

$$
g^{2}\left(\frac{\partial^{2} \psi}{\partial u^{2}}+\frac{\partial^{2} \psi}{\partial v^{2}}\right)=h(u, v, \psi) \frac{\partial \psi}{\partial t},
$$

where

$$
\begin{aligned}
g^{2} & =\left(\frac{\partial u}{\partial x}\right)^{2}+\left(\frac{\partial v}{\partial x}\right)^{2} \\
& =\left(\frac{\partial u}{\partial y}\right)^{2}+\left(\frac{\partial v}{\partial y}\right)^{2} \\
& =g^{2}(u, v) .
\end{aligned}
$$


Thus, defining

$$
H(u, v, \psi)=h(u, v, \psi) / g^{2}(u, v) .
$$

we have

$$
\begin{aligned}
\frac{\partial^{2} \psi}{\partial x^{2}}+\frac{\partial^{2} \psi}{\partial y^{2}} & =h(x, y, \psi) \frac{\partial \psi}{\partial t} \\
\rightarrow \frac{\partial^{2} \psi}{\partial u^{2}}+\frac{\partial^{2} \psi}{\partial v^{2}} & =H(u, v, \psi) \frac{\partial \psi}{\partial t} .
\end{aligned}
$$

We may therefore first transform our region into a rectangle and then solve by the method of Sec. 3. It should be noted that when a non-rectangular region is to be solved, the iterative scheme of Sec. 3 is necessary, even in the linear case.

One simple example of this method is a transformation to polar coordinates. Let

$$
u+i v=\log (x+i y)
$$

or

$$
u=\log r \quad v=\varphi,
$$

where $r$ and $\varphi$ are the standard polar coordinate radius and angle. Equation (2.1) becomes

$$
\frac{\partial^{2} \theta}{\partial u^{2}}+\frac{\partial^{2} \theta}{\partial v^{2}}=e^{2 u} \alpha^{-2} \frac{\partial \theta}{\partial t} .
$$

The singularity near the origin $(u \rightarrow-\infty$ as $r \rightarrow 0)$ should be noted. This transformation actually carries a slit annulus into a rectangle. There are other more complicated transformations which will carry a whole circle into a rectangle. For instance,

$$
u+i v=\int_{0}^{x+i y}\left(1-w^{4}\right)^{-1 / 2} d w .
$$

For problems with an axis of symmetry, we adopt cylindrical coordinates as the basic point of departure. The Laplacian is

$$
\nabla^{2} \psi=\frac{\partial^{2} \psi}{\partial r^{2}}+\frac{1}{r} \frac{\partial \psi}{\partial r}+\frac{1}{r^{2}} \frac{\partial^{2} \psi}{\partial \varphi^{2}}+\frac{\partial^{2} \psi}{\partial z^{2}} .
$$

As we assume an axis of symmetry we may choose our coordinate system so $\psi$ is independent of $\varphi$. Let us set

$$
\omega=r^{1 / 2} \psi
$$

Thus Eq. (3.1) becomes

$$
\frac{\partial^{2} \omega}{\partial r^{2}}+\frac{\partial^{2} \omega}{\partial z^{2}}=h(r, z, \omega) \frac{\partial \omega}{\partial t}-\frac{\omega}{4 r^{2}} .
$$

From the point of view of numerical solution we are free to add a term involving only $\omega$ and independent of any derivatives, and the method of solution proposed in Sec. 3 is still applicable. It should be noted that the iterative scheme will have to be modified to include this term, and if the coefficient of $\omega$ at the advanced time contributed by the right-hand side of the difference equation analogue of (5.11) may change sign, we may 
no longer pick $\beta$ so that the error will change sign everywhere between the guessed and calculated values of $\alpha$, but we must in some regions extrapolate for a new guess of $\omega$, rather than interpolate throughout as we did in Sec. 3.

The boundary condition to be applied along the axis of symmetry is that

$$
\frac{d(\log \omega)}{d(\log r)}=\frac{1}{2}
$$

This condition ensures that $\psi$ will be finite on the axis.

For many problems with an axis of symmetry, cylindrical coordinates are not the most convenient. Other axial shapes may be treated by conformally mapping them into a rectangle. A sphere is conveniently treated by transforming (5.11) by (5.6) where we, of course, consider only the right half $r-z$ plane $(-\pi / 2 \leq v \leq \pi / 2)$. Thus for spherical coordinates we get

$$
\frac{\partial^{2} \omega}{\partial u^{2}}+\frac{\partial^{2} \omega}{\partial v^{2}}=e^{2 u} h(u, v, \omega) \frac{\partial \omega}{\partial t}-\frac{\omega}{4} .
$$

6. Numerical examples. In order to illustrate the method described above we programmed it for an IBM 704. Two sample calculations are described.

We set up initial conditions corresponding to the solution of the linear problem,

$$
\theta(x, y, t)=\sin (\pi x / l) \sin (\pi y / l) \exp \left(-2 \alpha^{2} \pi^{2} t / l^{2}\right) .
$$

We chose $\alpha^{2} \Delta t /(\Delta x)^{2}=1.02392228$ so that the amplitude would be diminished by a factor of $10^{-1 / 16}$ at each time step. This choice enables us to check the accuracy at many times without excessive labor in calculating the analytic values. We ran this calculation with mesh points $15^{\circ}$ apart $(11 \times 11$ interior points), and advanced it 320 time steps. It was found that over the range of 20 decades through which the solution passed, the only discernible numerical error was a truncation error (the 704 does not round, but truncates instead) that caused the solution to decrease by an additional factor of $\left(1-5.44 \times 10^{-8}\right)$ at each time step. When a fixed number of decimal places are carried, the truncation error is expected to be proportional to $\alpha^{4}(\Delta t)^{2} /\left[(\Delta x)^{2}(\Delta y)^{2}\right]$. The difference between the solution of the difference equation and the differential equation is that time flows 2.5 per cent more rapidly in the latter case. It should be noted that the explicit scheme is unstable for this case. The calculating time for this case was about 6 minutes, or about 10 milliseconds per cycle point.

For our other example we chose radiation flow in a material medium. The equation is

$$
\nabla^{2}\left(\theta^{4}\right)=16 K \frac{\partial \theta}{\partial t} .
$$

If we let $\psi=K^{-4 / 3} \theta^{4},(6.2)$ becomes

$$
\nabla^{2} \psi=4 \psi^{-3 / 4} \frac{\partial \psi}{\partial t} .
$$

[If there were a power-law, temperature-dependent opacity, the equation would be

$$
\nabla \cdot\left(\theta^{i} \nabla \theta^{4}\right)=16 K \frac{\partial \theta}{\partial t} .
$$


By use of the identity

$$
\theta^{m} \nabla \theta^{n}=\frac{n}{n+m} \nabla \theta^{m+n}
$$

and the transformation

$$
\psi=\theta^{4+i}
$$

we can put (6.4) in the form of (3.1).]

For our sample calculation we picked $\beta(\Delta x)^{2}=\beta(\Delta y)^{2}=-1.5$ and used $11 \times 11$ interior mesh points. The other constants were chosen so that this is an optimal iteration, as defined by (3.3). For initial conditions we chose

$$
\psi(x, y)=1-\sin (2 \pi x / l) \sin (2 \pi y / l)
$$

and we maintained the boundaries at unit temperature. We added 0.01 to $\psi$ at the central point to prevent it from vanishing. Initially some 10 or 12 iterations were required per time step, but after 15 time steps, only about 2 iterations per time step were required for three-place accuracy. We make the initial guess of $\psi$ at each time step by a linear extrapolation from the two previous times. The running time for this calculation was about 15 minutes for 120 time steps, or about 20 milliseconds per cycle point. From runs with other values of $\beta(\Delta x)^{2}$ we estimate that the accuracy of this solution is about 2 to 3 per cent.

It should be noted that some care must be exercised to prevent the guessing of a negative temperature, as the occurrence of temperatures of different signs produces an instability which causes the solution to diverge.

\section{REFERENCES}

1. J. Douglas, Jr. and D. W. Peaceman, Numerical solution of two-dimensional heat-flow problems, A. I. Ch. E. J. 1, 505-512 (1955)

2. G. H. Bruce, D. W. Peaceman, H. H. Rachford, Jr. and J. D. Rice, Calculation of unsteady-state gas flow through porous media, Trans. Am. Inst. Mining Met. Engrs. 198, 79-92 (1953)

3. R. Bellman, $O n$ the weak and strong stability of numerical solutions of partial differential equations. 1 . The heat equation, Princeton University Rept. AECU-3275 (1958)

4. G. Birkhoff and S. MacLane, A survey of modern algebra, chap. IX, sec. 9, The Macmillan Co., New York, 1951

5. C. Caratheodory, Conformal representations, chap. V, University Press, Cambridge, 1932

6. E. T. Copson, Introduction to the theory of functions of a complex variable, chap. VIII, Clarendon Press, Oxford, 1948 